05,13

\title{
Магнитооптические, оптические и магнитотранспортные свойства сверхрешеток Со/Сu с ультратонкими слоями кобальта
}

\author{
() И.Д. Лобов, М.М. Кириллова, А.А. Махнев, Л.Н. Ромашев, А.В. Королев, \\ М.А. Миляев, В.В. Проглядо, Н.С. Банникова, В.В. Устинов
}

Институт фризики металлов им. М.Н. Михеева УрО РАН,

Екатеринбург, Россия

E-mail: i_lobov@imp.uran.ru

(Поступила в Редакцию 27 апреля 2016 г.)

Исследованы полевые зависимости намагниченности и магнитосопротивления сверхрешеток $\left[\mathrm{Co}\left(t_{x}, \AA\right) / \mathrm{Cu}(9.6 \AA)\right]_{30}$, приготовленных магнетронным распылением и отличающихся толщиной слоев Со $\left(0.3 \AA \leq t_{\text {Со }} \leq 15 \AA\right)$. Изучены оптические и магнитооптические свойства этих объектов методом эллипсометрии в области спектра $\hbar \omega=0.09-6.2 \mathrm{eV}$ и с помощью экваториального эффекта Керра $(\hbar \omega=0.5-6.2 \mathrm{eV})$. На кривых недиагональной компоненты тензора оптической проводимости $\hat{\sigma}$ сверхрешеток с $t_{\text {Со }}=3-15 \AA$ в ультрафиолетовой области обнаружена структура осцилляционного типа („петля“") - как результат обменного расщепления $3 d$-зоны в энергетическом спектре $f c c$ Co. На основе магнитных измерений и измерений экваториального эффекта Керра установлено наличие суперпарамагнитной фазы в сверхрешетках $\mathrm{Co} / \mathrm{Cu}$ при толщине слоев кобальта 3 и $2 \AA$. Анализируется переход от сверхрешеток со сплошными ферромагнитными слоями к кластерно-слоистым суперпарамагнитным наноструктурам и далее - к структурам на основе Со и $\mathrm{Cu}\left(t_{\mathrm{Co}}=0.3-1 \AA\right)$ с кондоподобным поведением электросопротивления при низких температурах.

Работа выполнена в рамках государственного задания ФАНО России (тема „Спин“, № 01201463330) при частичной финансовой поддержке РФФИ проект № 16-02-00061-a, программы УрО РАН „Фундаментальные основы технологий наноструктур и наноматериалов“, проект № 15-9-2-22, Минобрнауки РФ (грант № 14.Z50.31.0025).

DOI: 10.21883/FTT.2017.01.43950.161

\section{1. Введение}

Известно, что сверхрешетки $\mathrm{Co} / \mathrm{Cu}$ со сплошными ферромагнитными (FM) слоями Со обладают гигантским магнитосопротивлением (ГМС), величина которого при комнатной температуре может достигать значений $50-80 \%[1,2]$. Описание эффекта ГМС в наноструктурах $\mathrm{Co} / \mathrm{Cu}$ в рамках первопринципных вычислений для CIP (ток в плоскости пленки) и СРР (ток перпендикулярен плоскости пленки) - геометрий эксперимента проведено, в частности, в работах $[3,4]$ с учетом спиновой поляризации энергетических зон и $(s, p-d)$-гибридизации электронных состояний в $f c c$ Со. Числовые оценки коэффициента спиновой асимметрии рассеяния электронов проводимости на интерфейсе $\mathrm{Co} / \mathrm{Cu}$ получены из измерений ГМС в геометрии СРР $[5,6]$, из измерений ИК-магниторефрактивного эффекта [7], а также из первопринципных расчетов [8].

Мультислойные пленки с ультратонкими слоями FMметалла обладают уникальными магнитными и транспортными свойствами и привлекают внимание исследователей в связи с перспективой создания на их основе сенсорных устройств для слабых магнитных полей. Обнадеживающие результаты в этом направлении получены на образцах с кластерными слоями Fе и Сo (см., например, $[9,10])$. В отличие от слоевых пленок такие „квазигранулированные“ (кластерно-слоистые) пленки имеют изотропный эффект ГМС, пренебрежимо малый гистерезис намагниченности и показывают суперпарамагнитное (SPM) поведение. Дальнейшие исследования магнитных и магнитотранспортных свойств $\mathrm{Co} / \mathrm{Cu}$ показали, что толщина слоев $\mathrm{Co}$, способы и условия напыления образцов существенно влияют на формирование и свойства „квазигранулированных“ пленок [11-15].

Объектами исследования в настоящей работе являются мультислойные наноструктуры $\mathrm{Co}\left(t_{x}, \AA\right) / \mathrm{Cu}(9.6 \AA)$ $\left(0.3 \AA \leq t_{\text {Со }} \leq 15 \AA\right)$, обладающие гигантским магнитосопротивлением. Толщина слоя меди $t_{\mathrm{Cu}}=9.6 \AA$ соответствует области первого максимума межслоевого обменного взаимодействия антиферромагнитного (AF) типа. Нами изучены магнитные, магниторезистивные, магнитооптические, оптические и электрические свойства этих объектов. Целью работы является получение сведений об электронном энергетическом спектре и параметрах электронов проводимости сверхрешеток. Обсуждается также переход от сверхрешеток $\mathrm{Co}\left(t_{x}\right) / \mathrm{Cu}$ со сплошными FM слоями Со к кластерно-слоистым суперпарамагнитным наноструктурам и далее - к среде, в которой включения Со играют роль примесных центров рассеяния электронов в матрице меди.

\section{2. Образцы и методика эксперимента}

Сверхрешетки $\mathrm{Co} / \mathrm{Cu}$ были получены на магнетронной установке MPS-4000-C6 (ULVAC). В качестве подложек 
использовались монокристаллические пластины сапфира $(10 \overline{1} 2) \mathrm{Al}_{2} \mathrm{O}_{3}$ размером $30 \times 30 \times 0.5 \mathrm{~mm}$. Температура подложек во время напыления сверхрешеток поддерживалась комнатной, мощность магнетронного испарителя была $100 \mathrm{~W}$, давление аргона равнялось $0.1 \mathrm{~Pa}$.

Приготовлена серия сверхрешеток $\mathrm{Al}_{2} \mathrm{O}_{3} / \mathrm{Cr}(80 \AA) /$ $\left[\mathrm{Co}\left(t_{x}, \AA\right) / \mathrm{Cu}(9.6 \AA)\right]_{30} / \operatorname{Cr}(20 \AA)$, содержащих по 30 пар слоев $\mathrm{Co} / \mathrm{Cu}$ и отличающихся только номинальной тол-

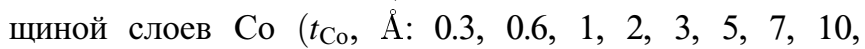
$15 \AA)$. Здесь и далее в тексте и на рисунках указаны номинальные толщины слоев, определенные по скорости и времени напыления. Скорости напыления слоев Со и $\mathrm{Cu}$ составляли соответственно 30 и $70 \AA / \mathrm{min}$. Аттестация образцов, проведенная при комнатной температуре методом малоугловой рентгеновской дифракции с использованием $\mathrm{Cu} K_{\alpha}$ линии излучения на рентгеновском дифрактометре Philips Empyrean, показала хорошую периодичность изученных мультислойных структур.

Магнитные свойства сверхрешеток исследовались при комнатной температуре с помощью вибрационного магнитометра в постоянных магнитных полях до $18 \mathrm{kOe}$, а также на СКВИД-магнитометре MPMS-XL 5 (Quantum Design) в полях до $50 \mathrm{kOe}$. Электросопротивление исследуемых наноструктур измеряли четырехконтактным методом в электромагните, а также на установке PPMS-9 (Quantum Design) в магнитном поле напряженностью до $90 \mathrm{kOe}$ в интервале температур 4.2-300 К. Измерение магнитосопротивления выполнялось при комнатной температуре в магнитном поле напряженностью до $32 \mathrm{kOe,} \mathrm{прикладываемом} \mathrm{вдоль} \mathrm{длинной} \mathrm{стороны}$ исследуемого образца, имеющего размеры $2.5 \times 12 \mathrm{~mm}$.

Измерения эффективных коэффициентов преломления $n^{\text {eff }}$ и поглощения $k^{\text {eff }}$ выполнены эллипсометрическим методом Битти в области длин волн $\lambda=0.2-14 \mu \mathrm{m}$. У всех образцов суммарная толщина мультислойной наноструктуры превышала глубину проникновения световой волны $\delta_{0}=c \omega / k^{\mathrm{eff}}(\omega-$ циклическая частота световой волны, $c-$ скорость света в вакууме). Измерения магнитооптического экваториального эффекта Керра (ЭЭК, $\delta_{p}$-эффект) выполнены в спектральном диапазоне $\hbar \omega=0.5-6.2 \mathrm{eV}$ $(\lambda=2.48-0.2 \mu \mathrm{m})$ в магнитном поле $H=4.9 \mathrm{kOe}$ при комнатной температуре и углах падения света на образец $\varphi$, равных 50 и $70^{\circ}$ по отношению к нормали к поверхности образца. Спектральные измерения в ультрафиолетовой (УФ) области $\hbar \omega=5-6.2 \mathrm{eV}$ проводились с использованием спектральной дуговой дейтериевой лампы ДДС 30.

\section{3. Магнитные и магниторезистивные свойства}

Рассмотрим изменение магнитных свойств сверхрешеток $\mathrm{Co}\left(t_{x}\right) / \mathrm{Cu}$ при уменьшении толщины слоев Co от 15 до $0.3 \AA$ и сохранении одинаковой у всех сверхрешеток толщины слоев $\mathrm{Cu}$. Исследования намагниченности показали, что при толщинах $t_{\text {Со }}=5-15 \AA$ сверх-
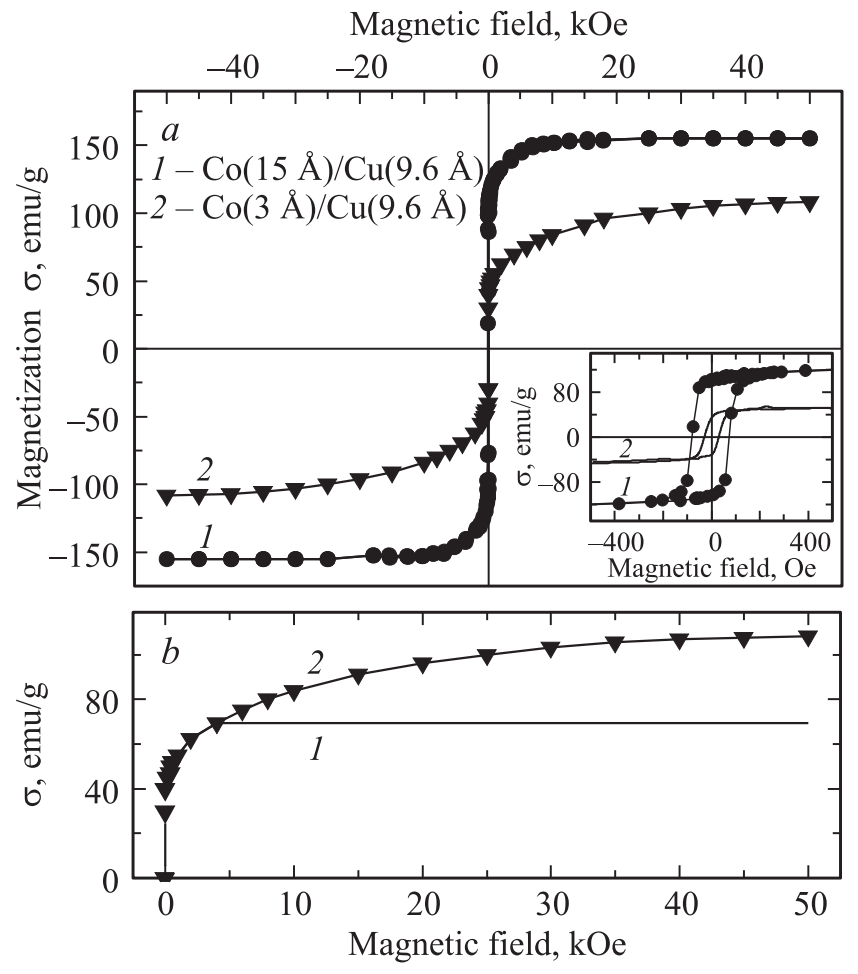

Рис. 1. Полевые зависимости намагниченности и петли магнитного гистерезиса (на вставке) сверхрешеток $\mathrm{Co}\left(t_{x}, \AA\right) / \mathrm{Cu}(9.6 \AA)\left(t_{x}, \AA: 1-15,2-3\right)$ при $T=300 \mathrm{~K}(a)$, 1 - FM вклад в намагниченность и 2 - суммарная $(\mathrm{FM}+\mathrm{SPM})$ намагниченность сверхрешетки с $t_{\mathrm{Co}}=3 \AA(b)$.

решетки обладают ферромагнитными свойствами. Для них характерно достижение при комнатной температуре намагниченности насыщения в сравнительно невысоких магнитных полях и наличие петель магнитного гистерезиса (см. рис. 1, $a$ ). Намагниченность сверхрешеток, имеющих толщину слоев Со от 3 до $5 \AA$, может быть представлена состоящей из FM и SPM частей. Схематически это показано на рис. $1, b$ для сверхрешетки с $t_{\text {Со }}=3 \AA$. Ферромагнитная часть намагниченности достигает магнитного насыщения в слабом магнитном поле, а суперпарамагнитная часть выделяется на кривой намагничивания в виде нелинейной функции, типичной для суперпарапроцесса. Наблюдаемый рост намагниченности (парапроцесс) обусловлен тем, что магнитные слои Со в толщинном интервале $3-5 \AA$ уже не являются сплошными, а состоят в значительной степени из отдельных ферромагнитных островков (кластеров), подобных тем, которые образуются на начальных стадиях формирования в сверхрешетках сплошных слоев Со. Напомним, что именно такие мультислойные наноструктуры, состоящие из сплошных слоев одного из металлов и кластерных слоев другого металла (в нашем случае Со), получили название кластерно-слоистых наноструктур.

Суперпарамагнитная часть кривой намагничивания кластерно-слоистой наноструктуры $\mathrm{Co} / \mathrm{Cu}$ с толщиной слоев Со $3 \AA$, представленная на рис. $1, b$, удовлетво- 


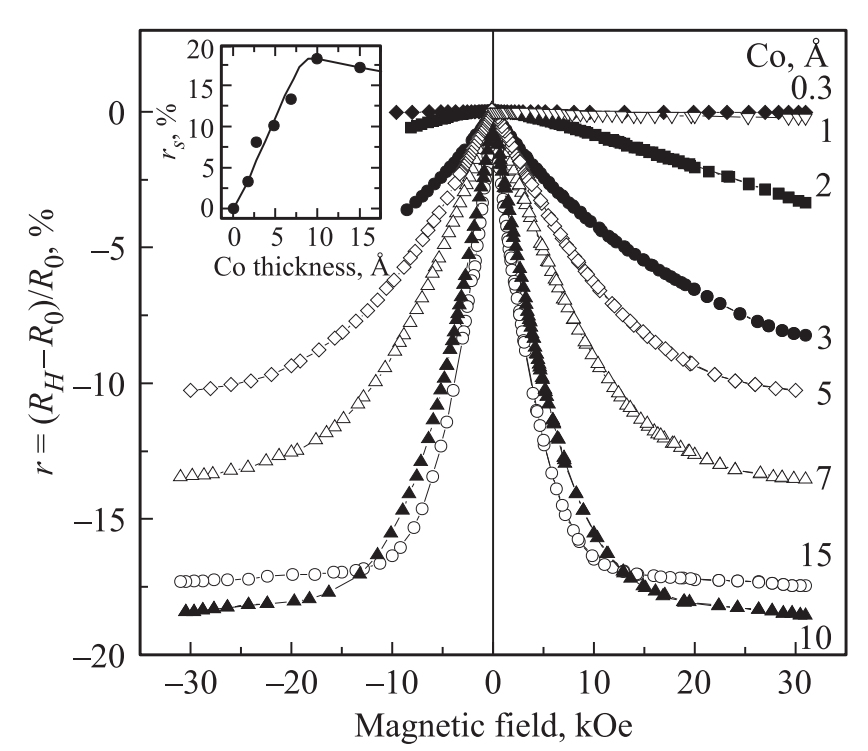

Рис. 2. Магнитосопротивление сверхрешеток $\mathrm{Co}\left(t_{x}, \AA\right) / \mathrm{Cu}(9.6 \AA)$ с различной толщиной слоев Со при $T=300 \mathrm{~K}$. На вставке: магнитосопротивление в магнитном поле $H=32 \mathrm{kOe}$.

рительно описывается функцией Ланжевена

$$
\frac{I}{I_{s}}=L\left(\frac{\mu H}{k T}\right)=\operatorname{cth} \frac{\mu H}{k T}-\frac{k T}{\mu H},
$$

где $I_{s}$ - намагниченность насыщения суперпарамагнитной части образца, $\mu$ - магнитный момент кластера, $H$ - напряженность магнитного поля, $T$ - темпеpaтура, $k-$ постоянная Больцмана. $I_{s}=N \mu, N-$ концентрация кластеров. Анализ кривых намагничивания сверхрешеток $\mathrm{Co} / \mathrm{Cu}$ с кластерными слоями $\mathrm{Co}$ показал, что средняя величина магнитных моментов кластеров составляет порядка 600 магнетонов Бора, что в пересчете на количество атомов соответствует $\sim 400$ атомам Со. С дальнейшим уменьшением толщины слоев $\mathrm{Co}\left(t_{\mathrm{Co}} \leq 2 \AA\right)$ сверхрешетки $\mathrm{Co} / \mathrm{Cu}$ при комнатной температуре приобретают магнитные свойства, характерные для идеальных суперпарамагнетиков, в которых кластеры имеют малые размеры.

Переходим к краткому рассмотрению магнитосопротивления сверхрешеток $\mathrm{Co} / \mathrm{Cu}$, отличающихся толщиной слоев Со. Полевые зависимости магнитосопротивления, измеренные при комнатной температуре в магнитном поле напряженностью до $32 \mathrm{kOe}$, приведены на рис. 2 . Видно, что по мере увеличения толщины слоев Со в сверхрешетках величина магнитосопротивления увеличивается, а величина поля насыщения уменьшается. Характерной особенностью полевых зависимостей магнитосопротивления сверхрешеток с тонкими и ультратонкими слоями Со является наличие у них протяженных линейных участков. У сверхрешеток со сплошными слоями Со при толщинах выше $5 \AA$ наблюдается выраженный переход к магнитному насыщению. На вставке на рис. 2 показано изменение величины ГМС сверхре- шеток с ростом толщины слоев Со. Приведенные на графике величины магнитосопротивления $r_{s}$ получены при напряженности магнитного поля, равной $32 \mathrm{kOe}$. Видно, что наибольшее значение магнитосопротивления у исследованной в данной работе серии мультислойных наноструктур наблюдается у сверхрешетки с $t_{\text {Со }}=10 \AA$.

\section{4. Экваториальный эфффект Керра}

Спектры экваториального эффекта Керра измерены при двух углах падения света на образец $(\varphi=50$ и $\left.\varphi=70^{\circ}\right)$ в поле $H=4.9 \mathrm{kOe}$. На рис. 3 приведены спектры ЭЭК при $\varphi=50^{\circ}$. Полевые зависимости $\delta_{p}(H)$ измерены при длине волны $\lambda=0.62 \mu \mathrm{m}$ и угле $\varphi=70^{\circ}$ в магнитных полях $H \leq 10 \mathrm{kOe}$ на поликристаллическом образце Со и сверхрешетках $\mathrm{Co}\left(t_{x}\right) / \mathrm{Cu}(9.6 \AA)$ с $t_{\mathrm{Co}}=2-15 \AA$. На образцах с толщинами $t_{\text {Со }}=0.3-1 \AA$ кривые $\delta_{p}(H)$ измерялись при угле $\varphi=50^{\circ}$ и $\lambda=0.4 \mu \mathrm{m}$. Измерения показали (рис. 4), что полевые зависимости ЭЭК образцов с толщинами слоев кобальта 5-15 зуют ход намагничивания в присутствии межслоевого обменного взаимодействия AF типа, проявляющегося на кривых $\delta_{p}(H)$ монотонным увеличением эффекта с ростом магнитного поля. Следует отметить четырехкратное различие в амплитудах ЭЭК образцов с толщинами $t_{\text {со }}$, равными 7 и $5 \AA$. Полевые зависимости $\delta_{p}(H)$ образцов с $t_{\text {Со }} \leq 3 \AA$ сушественно отличаются от кривых $\delta_{p}(H)$, полученных на образцах с толщинами $t_{\text {Со }}>3 \AA$. Во-первых, сменился знак эффекта во всем диапазоне магнитных полей. Во-вторых, полевые зависимости приобрели суперпарамагнитный характер из-за наличия вкладов FM и SPM фракций. В полях $H>H_{s}$, где $H_{s}$ - поле насыщения для FM фазы, ЭЭК можно представить в виде $\delta_{p}(H)=c_{f}+b L(\alpha H)$, где $c_{f}-\mathrm{FM}$ вклад в $\delta_{p}$-эффект, $b-$ парамагнитный

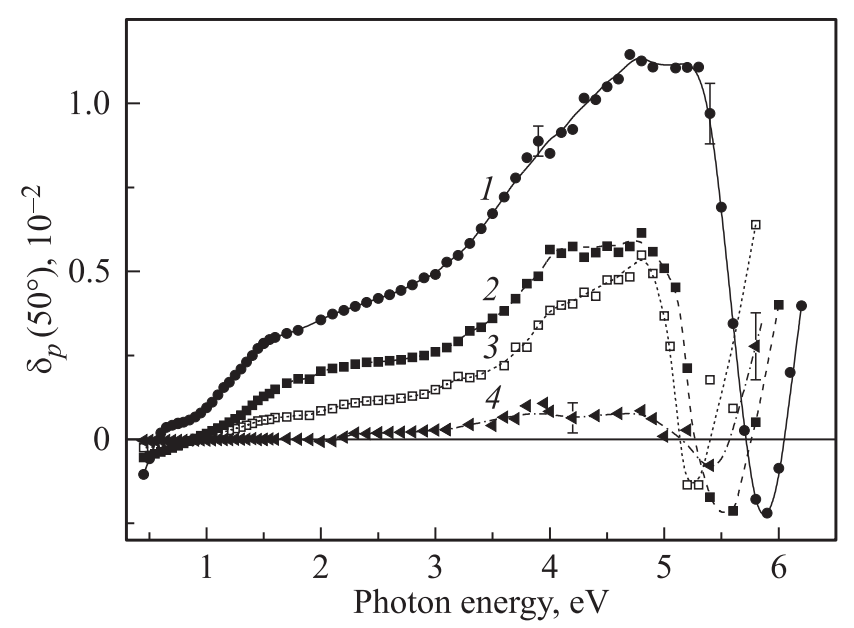

Рис. 3. Спектры ЭЭК объемного $h c p$ Со (1) и сверхрешеток $\mathrm{Co}\left(t_{x}, \AA\right) / \mathrm{Cu}(9.6 \AA)\left(t_{x}, \AA: 2-15,3-7,4-3\right)$ в магнитном поле $H=4.9 \mathrm{kOe}$ при угле падения света на образец $\varphi=50^{\circ}$. Вертикальными отрезками обозначены ошибки измерений. 
вклад, связанный с SPM состояниями и обеспечивающий монотонный рост эффекта с увеличением магнитного поля, $L(\alpha H)-$ функция Ланжевена (1). Для образца с $t_{\text {Со }}=3 \AA$ приведена численная аппроксимация полевой зависимости ЭЭК (штриховая кривая) функцией $L(\alpha H)$ в полях $H>1000$ Ое, при этом число атомов Со в SPM кластере, как и в случае анализа магнитных измерений, составляет $\sim 400$, а соотношение $\mathrm{FM}$ и SPM вкладов в $\delta_{p}$-эффект равняется 4:6. Полевые зависимости ЭЭК образцов с $t_{\mathrm{Co}}=0.3-2 \AA$ не имеют FM составляющей, что согласуется с данными магнитных измерений при комнатной температуре.

Можно предположить, что наблюдаемая в эксперименте смена знака $\delta_{p}$-эффекта при $t_{\text {Со }}=3 \AA$ обусловлена изменением структуры пленок от однородноплоскостной к гранулированной. В связи с этим нами выполнены расчеты толщинных зависимостей ЭЭК для $\mathrm{Co} / \mathrm{Cu}$ в плоскостной модели [16] и в модели эффективной среды [17] для гранулированного сплава. Кратко приведем схемы расчетов.

Плоскостная модель [16]. В многослойной структуре при экваториальном однородном намагничивании FM слоев и $p$-поляризации падающей световой волны эффект относительного изменения интенсивности отраженного света (ЭЭК) дается выражением

$$
\left(\frac{\Delta I}{I_{0}}\right)_{p}=\delta_{p}=\operatorname{Im} \sum_{j=1}^{N-1} R_{j}\left(1-F_{j}^{2}\right) \frac{n_{1} Q_{j}}{g_{j}} \sin \varphi \cos \gamma_{j}
$$

где $n_{1}$ - показатель преломления первой среды (обычно $\left.n_{1}=1\right), N-$ число слоев, $\varphi$ - угол падения света, $\gamma_{j}$ - угол, образуемый вектором намагниченности $j$-го слоя с нормалью к плоскости падения света (экваториальный угол намагниченности), $Q_{j}-$ магнитооптический параметр, пропорциональный намагниченности, $g_{j}=\sqrt{\varepsilon_{j}-\sin ^{2} \varphi}, \quad\left(\varepsilon_{j}-\right.$ диэлектрическая проницаемость слоя). Фазовый множитель $F_{j}=e\left(-2 \pi i g_{j} t_{j} / \lambda\right)$ определяет набег фазы световой волны $(\lambda-$ длина волны в вакууме) и ее затухание в слое с толщиной $t_{j}$. Коэффициенты $R_{j}$ являются известными функциями $\varepsilon_{j}$ и $F_{j}$. Суммирование в (2) ведется по слоям с $Q_{j} \neq 0$. При расчете ЭЭК многослойной структуры $\mathrm{Co} / \mathrm{Cu}$ по формуле (2) были использованы значения $\varepsilon_{\mathrm{Co}}, \varepsilon_{\mathrm{Cu}}$ и $Q_{\mathrm{Co}}$, полученные нами из измерений на поликристаллическом образце Со и пленке меди ( $1000 \AA)$.

4.1. Модель эффективной среды для гранулированного сплава [17]. Пусть

$$
\widehat{\varepsilon}_{m}=\left(\begin{array}{ccc}
\varepsilon_{1} & i \gamma_{1} & 0 \\
-i \gamma_{1} & \varepsilon_{1} & 0 \\
0 & 0 & \varepsilon_{1}
\end{array}\right), \quad \widehat{\varepsilon}_{0}=\left(\begin{array}{ccc}
\varepsilon_{0} & 0 & 0 \\
0 & \varepsilon_{0} & 0 \\
0 & 0 & \varepsilon_{0}
\end{array}\right)
$$

- тензоры диэлектрической проницаемости ферромагнитной среды $\left(\widehat{\varepsilon}_{m}\right)$ и немагнитной матрицы $\left(\widehat{\varepsilon}_{0}\right)$. Магнитооптические свойства гранулированного сплава описываются тензором эффективной диэлектрической проницаемости $\widehat{\varepsilon}_{l}(\omega)$. В линейном по намагниченности приближении (Н параллельно оси $O Z) \widehat{\varepsilon}_{l}$ может быть представлен в виде

$$
\widehat{\varepsilon}_{l}=\left(\begin{array}{ccc}
\varepsilon_{l} & i \gamma_{l} & 0 \\
-i \gamma_{l} & \varepsilon_{l} & 0 \\
0 & 0 & \varepsilon_{l}
\end{array}\right)
$$

Диагональная $\varepsilon_{l}$ и недиагональная $\gamma_{l}$ компоненты тензора находятся из системы уравнений

$$
\left\{\begin{array}{l}
\frac{q\left(\varepsilon_{1}-\varepsilon_{l}\right)}{\varepsilon_{l}+\frac{1}{2}\left(1-L_{0}\right)\left(\varepsilon_{1}-\varepsilon_{l}\right)}+\frac{(1-q)\left(\varepsilon_{0}-\varepsilon_{l}\right)}{\varepsilon_{l}+\frac{1}{2}\left(1-L_{0}\right)\left(\varepsilon_{0}-\varepsilon_{l}\right)}=0, \\
\frac{q\left(\gamma_{l}-\gamma_{1}\right)}{\left[\varepsilon_{l}+\frac{1}{2}\left(1-L_{0}\right)\left(\varepsilon_{1}-\varepsilon_{l}\right)\right]^{2}}+\frac{(1-q) \gamma_{l}}{\left[\varepsilon_{l}+\frac{1}{2}\left(1-L_{0}\right)\left(\varepsilon_{0}-\varepsilon_{l}\right)\right]^{2}}=0 .
\end{array}\right.
$$

Здесь $q$ - объемное (в атомных \%) содержание FM фракции, $L_{0}-$ фактор формы гранул (для сферических частиц $\left.L_{0}=1 / 3\right)$. В рамках этого приближения ЭЭК для массивного гранулированного сплава можно найти по формуле [18,19]

$$
\left(\frac{\Delta I}{I_{0}}\right)_{p}=\delta_{p}=-4 \operatorname{tg} \varphi \operatorname{Im}\left[\frac{\gamma_{l} \operatorname{tg} \varphi \cos \gamma}{\left(\varepsilon_{l}-1\right)\left(\varepsilon_{l}-\operatorname{tg}^{2} \varphi\right)}\right],
$$

где $\gamma$ - экваториальный угол намагничивания образца и $\varphi$ - угол падения света. Предполагается, что магнитные гранулы одинаковы по величине и регулярно расположены по объему матрицы. Полученные нами данные об оптических и магнитных свойствах многослойных структур $\mathrm{Co} / \mathrm{Cu}$ дают основание предполагать, что при переходе к тонким слоям Со $\left(t_{\text {Со }}<7 \AA\right)$ слоистая структура $\mathrm{Co} / \mathrm{Cu}$ трансформируется в гранулированный сплав, состоящий из изолированных частиц Со (магнитных кластеров), внедренных в матрицу меди. Имеем $\varepsilon_{0}=\varepsilon_{\mathrm{Cu}}$,

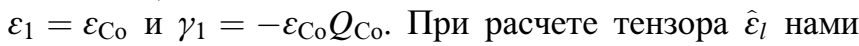
использованы те же значения объемных проницаемостей, что и при расчете ЭЭК в слоистой модели. Поскольку постоянные $f c c$ решеток $\mathrm{Co} \mathrm{и} \mathrm{Cu}$ близки $(\sim 3.61 \AA)$, то параметр $q$ (объемная концентрация атомов Со в образце) можно определить выражением $q=t_{\mathrm{Co}} /\left(t_{\mathrm{Co}}+t_{\mathrm{Cu}}\right)$, где $t_{\mathrm{Co}}$ и $t_{\mathrm{Cu}}-$ номинальные толщины слоев.

Из рис. 4 видно, что при переходе от $t_{\text {Со }}=5 \AA$ к $t_{\text {Со }}=3 \AA$ величина эффекта не уменьшилась, но произошла смена знака ЭЭК. На вставке к рисунку представлены расчетные кривые толщинных зависимостей ЭЭК в многослойной (кривая 1) и гранулярной $\left(L_{0}=1 / 3\right.$ и $2 / 3, q=0.238$, кривая 2) схемах, а также экспериментальная кривая $\delta_{p}\left(t_{\mathrm{Co}}\right)$, кривая 3. Моделирование в слоистой, гранулярной и в слоистогранулярной (учет тонкого слоя магнитного интерфейса $\mathrm{CoCu}$ в виде гранул Со в матрице меди производится в гранулярной схеме и используется далее в плоскостной 


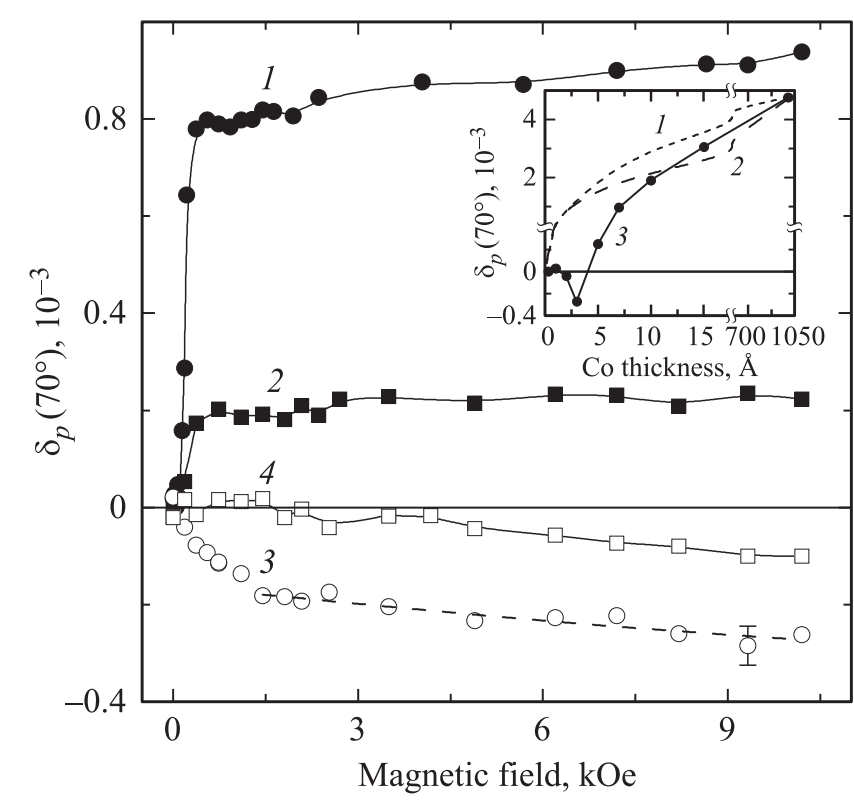

Рис. 4. Полевые зависимости ЭЭК сверхрешеток $\mathrm{Co}\left(t_{x}, \AA\right) / \mathrm{Cu}(9.6 \AA) \quad\left(t_{x}, \AA ̊ 丿: 1-7,2-5,3-3,4-2\right)$ при угле падения света на образец $\varphi=70^{\circ}$ и длине волны $\lambda=0.62 \mu \mathrm{m}$. Штриховая линия на кривой (3) - численная аппроксимация полевой зависимости $\delta_{p}\left(70^{\circ}\right)$ функцией $L(a H)$ в полях $H>1 \mathrm{kOe}$. На вставке: $1-$ расчет $\delta_{p}\left(70^{\circ}\right)$ в модели слоистой среды, $2-$ расчет $\delta_{p}\left(70^{\circ}\right)$ в модели гранулярной среды, 3 - эксперимент $\delta_{p}\left(70^{\circ}\right)$.

схеме расчета $\mathrm{Cu} / \mathrm{CoCu} / \mathrm{Co} / \mathrm{CoCu}$ ) моделях не объясняет смену знака ЭЭК, наблюдаемую экспериментально при толщине слоя кобальта в бислое $t_{\text {со }}=3 \AA$. Дальнейший численный анализ показал, что сдвиг смены знака ЭЭК от $\sim 1 \mathrm{eV}$ к энергии фотона $\sim 2 \mathrm{eV}$ происходит при специфическом наборе параметров $\left(L_{0} \sim 1, q<0.1\right)$ в гранулярной схеме. Значение $L_{0} \sim 1$ означает представление гранул Со в виде тонких пластинок в плоскости пленки, а малые значения параметра $q$ свидетельствуют о значительном перераспределении атомов кобальта между FM, SPM и парамагнитной фракциями кобальта в пользу последней. Отметим, что в работе [20] наблюдали смену знака ЭЭК в однородных сплавах $\mathrm{CoCu}$.

\section{5. Оптические и магнитооптические свойства}

5.1. Электронные характеристики $\mathrm{Co} / \mathrm{Cu}$. Диагональную компоненту тензора $\hat{\varepsilon}$ запишем в виде $\varepsilon_{x x}=\varepsilon^{\prime}-i \varepsilon^{\prime \prime}$. На рис. 5, $a, b$ приведены графики спектральной зависимости функций $\varepsilon^{\prime}(\lambda)$ и $\varepsilon^{\prime \prime}(\lambda)$ для исследованных нами образцов $\mathrm{Co}\left(t_{x}, \AA\right) / \mathrm{Cu}(9.6 \AA)$. Друдевский характер поведения этих величин в ИК-области спектра позволяет оценить параметры электронов проводимости - эффективные плазменную $\hbar \omega_{p}$ и релаксационную $\gamma_{\text {opt }}$ частоты и проследить за их изменением с уменьшением толщины слоя кобальта. Значения указан- ных параметров получены нами из наклона прямолинейного участка графика $1 /\left(1-\varepsilon^{\prime}\right)=\omega^{2} / \omega_{p}^{2}+\gamma_{\text {opt }}^{2} / \omega_{p}^{2}$ и отрезка, отсекаемого этой прямой на оси ординат (вкладом от виртуальных электронных переходов пренебрегали). Спектральные интервалы, использованные нами для построения зависимости $1 /\left(1-\varepsilon^{\prime}\right)=f\left(\omega^{2}\right)$, изменялись в пределах длины волны $\lambda$ от 2.5 до $14 \mu \mathrm{m}$ для выполнения условия $D \geq \delta_{0}(D-$ общая толщина слоистой пленки, $\delta_{0}-$ глубина проникновения световой волны). Полученные данные приведены на рис. 6. Можно констатировать, что плазменная частота $\hbar \omega_{p}$, пропорциональная числу свободных электронов в эффективной среде, возрастает с уменьшением фракции кобальта, приближаясь к своему значению в чистой меди $\left(\hbar \omega_{p}=8.5 \mathrm{eV}\right)$. Параметр $\tau_{\mathrm{opt}}^{\text {eff }}$ на участке толщин $0.3 \AA \leq t_{\text {Со }} \leq 15 \AA$ характеризует суммарное рассеяние электронов проводимости в объеме слоев $\mathrm{Cu}, \mathrm{Co}$, кластеров кобальта, а также на интерфейсах. Отметим, что сверхрешетки с толщинами $t_{\text {Со }}$, равными 15 и $10 \AA$ имеют значения $\tau_{\mathrm{opt}}^{\mathrm{eff}}$ и $\hbar \omega_{p}^{\mathrm{eff}}$, аналогичные полученным нами ранее [7]. Анализ толщинной зависимости времени релаксации электронов проводимости $\tau_{\mathrm{opt}}^{\mathrm{eff}}\left(t_{\mathrm{Co}}\right)$ (рис. 6)

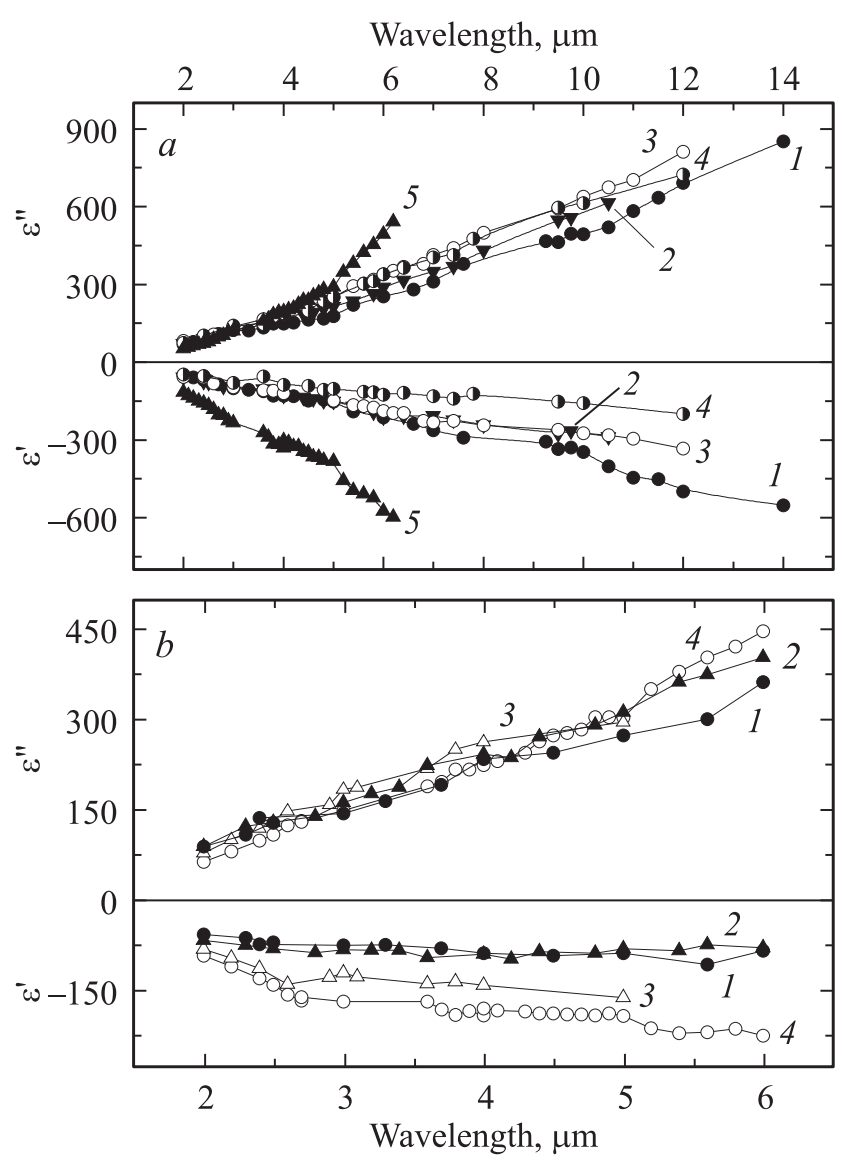

Pис. 5. $a, b$ Спектральные зависимости действительной $\varepsilon^{\prime}(\lambda)$ и мнимой $\varepsilon^{\prime \prime}(\lambda)$ частей комплексной диэлектрической проницаемости сверхрешеток $\mathrm{Co}\left(t_{x}, \AA\right) / \mathrm{Cu}(9.6 \AA) . a-\left(t_{x}, \AA: 1-15\right.$, $2-10,3-7,4-5,5-0.3), b-\left(t_{x}, \AA: 1-3,2-2\right.$, $3-1,4-0.6)$. 


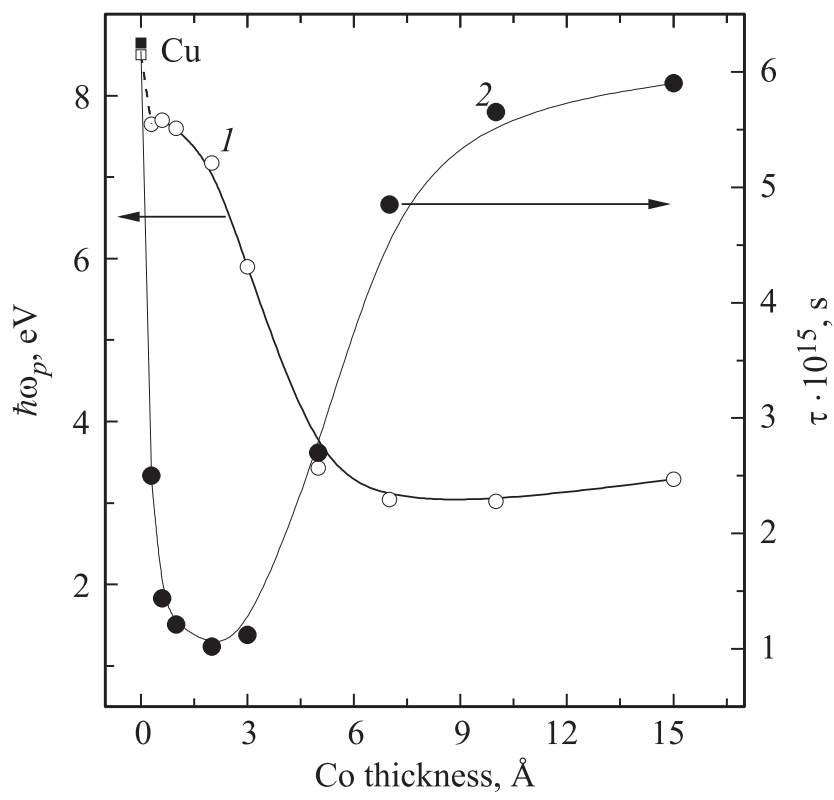

Рис. 6. Зависимости параметров электронов проводимости сверхрешеток $\mathrm{Co}\left(t_{x}, \AA\right) / \mathrm{Cu}(9.6 \AA)$ от толщины слоя $\mathrm{Co}: 1-$ плазменная частота $\hbar \omega_{p}^{\text {eff }}, 2-$ время релаксации $\tau_{\text {oft }}^{\text {eff. }}$ Графики дополнены данными $\hbar \omega_{p}$ (светлый квадрат) и $\tau_{\text {орt }}$ (темный квадрат) для пленки $\mathrm{Cu}$.

позволяет заключить, что кластеризация слоев кобальта начинается при $t_{\text {Со }}<7 \AA$. Об этом свидетельствует как начавшийся резкий спад величины $\tau_{\text {opt }}^{\text {eff }}$, так и четырехкратное уменьшение амплитуды $\delta_{p}$-эффекта в образце с $t_{\text {Со }}=5 \AA$ по сравнению с наблюдаемой величиной ЭЭК в пленке $\mathrm{Co}(7 \AA) / \mathrm{Cu}(9.6 \AA)$ (рис. 4). Сформировавшаяся кластерно-слоистая структура в $\mathrm{Co}(2 \AA) / \mathrm{Cu}(9.6 \AA)$ имеет минимальное значение $\tau_{\mathrm{opt}}^{\text {eff }}=1.02 \cdot 10^{-15} \mathrm{~s}$. Рост функции $\tau_{\mathrm{opt}}^{\mathrm{eff}}\left(t_{\mathrm{Co}}\right)$ при толщинах слоя кобальта $1,0.6$, и $0.3 \AA$ указывает на уменьшение рассеяния электронов проводимости в $\mathrm{Co} / \mathrm{Cu}$. Это связано с дальнейшим уменьшенном размеров интерфейсных и кобальтовых включений (кластеров) и понижением их концентрации в матрице меди.

5.2. Диагональная $\sigma_{x x}$ и недиагональная $\sigma_{x y}$ компоненты тензора оптической проводимости $\hat{\sigma}$. Связь компонент тензора диэлектрической проницаемости $\hat{\varepsilon}$ с соответствующими компонентами тензора оптической проводимости $\hat{\sigma}$ (ось $O Z$ параллельна Н) описывается выражениями

$$
\varepsilon_{x x}=1-\frac{4 \pi i}{\omega} \sigma_{x x}, \quad \varepsilon_{x y}=-\frac{4 \pi i}{\omega} \sigma_{x y} .
$$

В рамках модели эффективной среды можно пользоваться обычными формулами для магнитооптических эффектов. В частности, при расчете недиагональной компоненты $\sigma_{x y}$ тензора оптической проводимости $\hat{\sigma}$ сверхрешеток мы использовали выражение (6) для экваториального эффекта Керра при р-поляризации падающей электромагнитной волны в магнитном поле, приложенном перпендикулярно плоскости падения света. При $\mathbf{M} \|$ [001] (M - вектор намагниченности) поглощение световой энергии описывается функциями $\operatorname{Re} \sigma_{x x}(\omega)$ и $\operatorname{Im} \sigma_{x y}(\omega)$. Частотные зависимости функции $\omega \operatorname{Im} \sigma_{x y}(\omega)$ сверхрешеток $\mathrm{Co} / \mathrm{Cu}$, рассчитанные из значений $\delta_{p}$ при двух углах падения световой волны и эффективных оптических постоянных $n^{\text {eff }}$ и $k^{\mathrm{eff}}$, приведены на рис. 7. Графики оптической проводимости $\operatorname{Re} \sigma_{x x}(\omega)$ исследуемых пленок представлены на рис. 8. Проанализируем эти результаты с позиций электронной структуры, опираясь на имеющиеся теоретические вычисления.

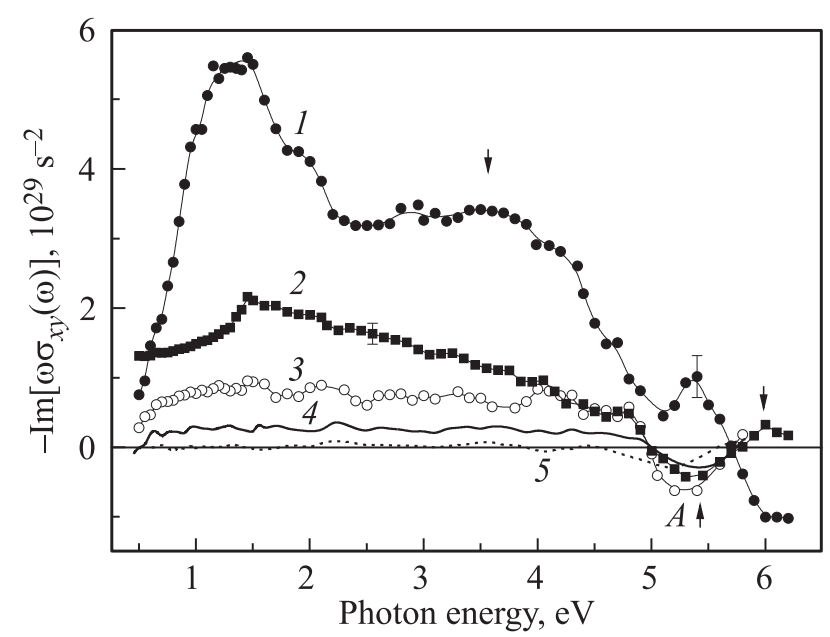

Рис. 7. Частотная зависимость функции $\omega \operatorname{Im} \sigma_{x y}(\omega)$ массивного $h c p$ Со $(1)$ и сверхрешеток $\mathrm{Co}\left(t_{x}, \AA\right) / \mathrm{Cu}(9.6 \AA)\left(t_{x}, \AA\right.$ : $2-15,3-7,4-3,5-2)$. Стрелками „ир“ и „down“ указаны области спектра с преобладающим по спину типом электронных переходов. Символом $A$ обозначена осцилляционная структура („петля“), обусловленная обменным расщеплением $3 d$-зоны $f c c$ Сo. Вертикальными отрезками обозначены ошибки определения функции.

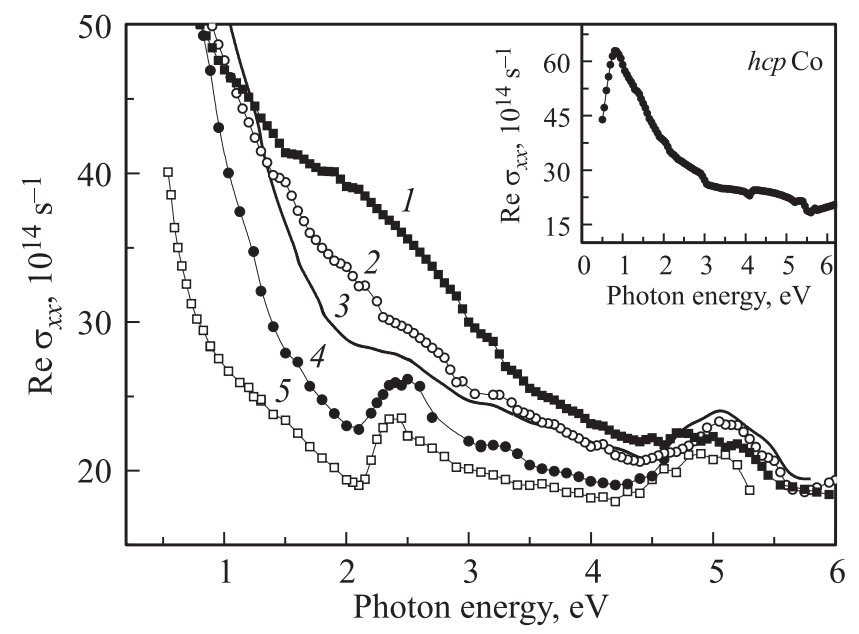

Рис. 8. Спектры диагональной компоненты тензора оптической проводимости $\operatorname{Re} \sigma_{x x}(\omega)$ сверхрешеток $\operatorname{Co}\left(t_{x}, \AA\right) / \mathrm{Cu}(9.6 \AA)$ $\left(t_{x}, \AA: 1-15,2-7,3-3,4-1,5-0.3\right)$. На вставке: функция $\operatorname{Re} \sigma_{x x}(\omega) h c p \operatorname{Co.}$ 
Численные расчеты тензора $\hat{\sigma}$ FM Co в $f c c$ (массивной) и $h c p$ фазах выполнены в релятивистском приближении методом LMTO (методом линеаризованных „маффин-тик“ орбиталей) в рамках теории функционала локальной электронной и спиновой плотности [21]. В обеих фазах расчеты предсказали формирование сдвоенного пика поглощения на кривой $\operatorname{Re} \sigma_{x x}(\omega)$ в УФ области $(\hbar \omega=5.0-6.5 \mathrm{eV})$ и как следствие - смену знака функции $\omega \operatorname{Im} \sigma_{x y}(\omega)$ и образование полосы осцилляционного типа („петли“). Сдвоенный пик в оптическом поглощении формируется вкладами межзонных переходов электронов со спинами по направлению $(\uparrow)$ и против направления $(\downarrow)$ спонтанной намагниченности.

Для проверки теоретических результатов нами дополнительно изучены оптические и магнитооптические свойства массивного поликристаллического Со в спектральной области, включающей УФ-участок спектра 5.0-6.2 eV (рис. 7, вставка на рис. 8). На экспериментальной кривой $\operatorname{Re} \sigma_{x x}(\omega)$ кобальта межзонные вклады электронов со спинами вверх $\uparrow$ и вниз $\downarrow$ неразличимы из-за лоренцевского уширения полос поглощения. Однако эта специфическая особенность в оптическом поглощении кардинально повлияла на магнитооптический отклик кобальта. Используя некоторые упрощающие предположения, авторы [21] получили соотношение, связывающее диагональную и недиагональную компоненты тензора $\hat{\sigma}$ в виде

$$
\operatorname{Im} \sigma_{x y}(\omega) \propto \operatorname{Re}\left[\sigma_{x x}^{\uparrow}(\omega)-\sigma_{x x}^{\downarrow}(\omega)\right],
$$

справедливое при энергиях фотона $2 \xi<\hbar \omega<W_{d}$ (2 $\xi$ - средняя величина спин-орбитального расщепления энергетических зон, $W_{d}$ - ширина $3 d$-зоны FM металла). В силу соотношения (8) частотная зависимость функции $\omega \operatorname{Im} \sigma_{x y}(\omega)$ становится знакопеременной, фиксируя на шкале энергий фотона области межзонных вкладов с преобладающим по спину электронов типом переходов. Полученные нами результаты по частотной зависимости функции $\omega \operatorname{Im} \sigma_{x y}(\omega)$ для поликристаллического Со с hcp решеткой (рис. 7, кривая 1) согласуются с теоретической кривой $\omega \operatorname{Im} \sigma_{x y}(\omega)$, приведенной в [21, рис.8]: смена знака при энергии $5.7 \mathrm{eV}$, „Петля“ в области $\hbar \omega=6.2 \mathrm{eV}$. Отметим, что смену знака $\omega \operatorname{Im} \sigma_{x y}(\omega)$ при энергии фотона $5.4 \mathrm{eV}$ в поликристаллическом $h c p$ Со ранее наблюдали в работе [22].

Обратимся к рассмотрению магнитооптического отклика в системе мультислойных наноструктур $\mathrm{Co}\left(t_{x}, \AA\right) / \mathrm{Cu}(9.6 \AA)$ (рис. 7). Присутствие слоев меди и начавшаяся при $t_{\text {Со }}<7 \AA$ кластеризация кобальта существенно повлияли на величину и частотную зависимость функции $-\omega \operatorname{Im} \sigma_{x y}(\omega)$. В частности, интенсивный пик при энергиях фотона $1-2 \mathrm{eV}$ слабо проявился в структуре $\mathrm{c} t_{\mathrm{Co}}=15 \AA$ и полностью отсутствует при толщинах кобальта 7 и $3 \AA$. Однако ключевая особенность, связанная с обменным расщеплением $3 d$-зоны в Co, а именно, „петля“ А в УФ-области спектра наблюдается в сверхрешетках и кластерно-слоистой пленке $\mathrm{Co} / \mathrm{Cu}$ при $t_{\text {Со }}=3-15 \AA$ (рис. 7, кривые 2-4). Главное отличие этих кривых от аналогичной функции $\omega \operatorname{Im} \sigma_{x y}(\omega) h c p$ Co (рис. 7, кривая 1) состоит в том, что точка пересечения с осью абсцисс, связанная с шириной $3 d$-зоны, сместилась к энергии фотона $5 \mathrm{eV}$. Соответственно к низким энергиям фотона сместилась и „петля“ $A$, обусловленная обменным расщеплением $3 d$-зоны в $f c c$ Co.

Этот экспериментальный результат имеет объяснение. Структурные исследования показали, что пленки кобальта толщиной $t_{\text {Со }} \leq 20 \AA$, напыленные на подложки $\mathrm{Cu}$, имеют напряженную $f c c$ структуру с латеральным параметром $a=3.59 \pm 0.02 \AA$ [23], либо $a=3.61 \pm 0.01 \AA[24]$, растянутым на $1.4-2 \%$ по сравнению с параметром кристаллической решетки массивного $f c c$ Co $(a=3.5447 \AA$ [25]). Увеличение параметра $a$ приводит к сужению $3 d$-зоны $f c c$ Со в мультислойных наноструктурах $\mathrm{Co} / \mathrm{Cu}$ и уменьшению обменного расщепления $3 d$-зоны, что подтверждают полученные нами результаты. Следуя схеме расчета [21], мы получили величину обменного расщепления $3 d$-зоны в энергетическом спектре $f c c$ Сo, равную $2 \Delta_{e x}^{3 d} \sim 1 \mathrm{eV}$. Таким образом, при комнатной температуре ферромагнитные свойства мультислойных наноструктур $\mathrm{Co}\left(t_{x}, \AA\right) / \mathrm{Cu}(9.6 \AA)$ сохраняются при толщинах слоя $t_{\mathrm{Co}}=3-15 \AA$. Как магнитные, так и магнитооптические данные показали, что в пленке $\mathrm{Co}(3 \AA) / \mathrm{Cu}(9.6 \AA)$ формируются две фракции: SPM и FM кластеры Со. Время релаксации электронов проводимости в такой пленке резко уменьшается до значения $\tau_{\mathrm{opt}}^{\mathrm{eff}}=1.21 \cdot 10^{-15} \mathrm{~s}$.

$\mathrm{B}$ образце $\mathrm{c} t_{\mathrm{Co}}=2 \AA$ происходит дальнейшая потеря магнитных свойств, о чем можно судить по падению величины намагниченности, отсутствию магнитного гистерезиса при комнатной температуре, а также по занулению функции $\operatorname{Im} \sigma_{x y}(\omega)$ в широком спектральном интервале $0.5-4.6 \mathrm{eV}$ (рис. 7, кривая 5). В этом образце наблюдается наиболее сильное рассеяние электронов проводимости $\left(\tau_{\mathrm{opt}}^{\mathrm{eff}}=1.02 \cdot 10^{-15} \mathrm{~s}\right)$.

\section{6. Кондоподобное поведение электросопротивления}

В разделе 5.1 было отмечено, что полученные при комнатной температуре оптические данные указывают на рост функции $\tau_{\text {opt }}^{\text {eff }}$ начиная с толщины слоя кобальта $t_{\text {Со }} \leq 1 \AA$. Это свидетельствует об уменьшении рассеяния электронов проводимости в кластерно-слоистых наноструктурах $\mathrm{Co} / \mathrm{Cu}$, связанном с дальнейшим уменьшением размеров интерфейсных и Со кластеров и понижением их концентрации в матрице меди. Таким образом, кластерно-слоистая наноструктура $\mathrm{Co} / \mathrm{Cu}$ оказалась эффективной средой, в которой включения Со начинают играть роль примесных центров рассеяния электронов проводимости в меди.

Выполненные нами температурные измерения электросопротивления $\rho(T) \quad(T=4.2-300 \mathrm{~K})$ на сверхрешетках $\mathrm{Co} / \mathrm{Cu}$ с ультратонкими слоями кобальта $t_{\text {Со }} \leq 1 \AA$ показали (рис. 9, $a$ ), что в этих структурах наблюдается аномалия температурной зависимости 


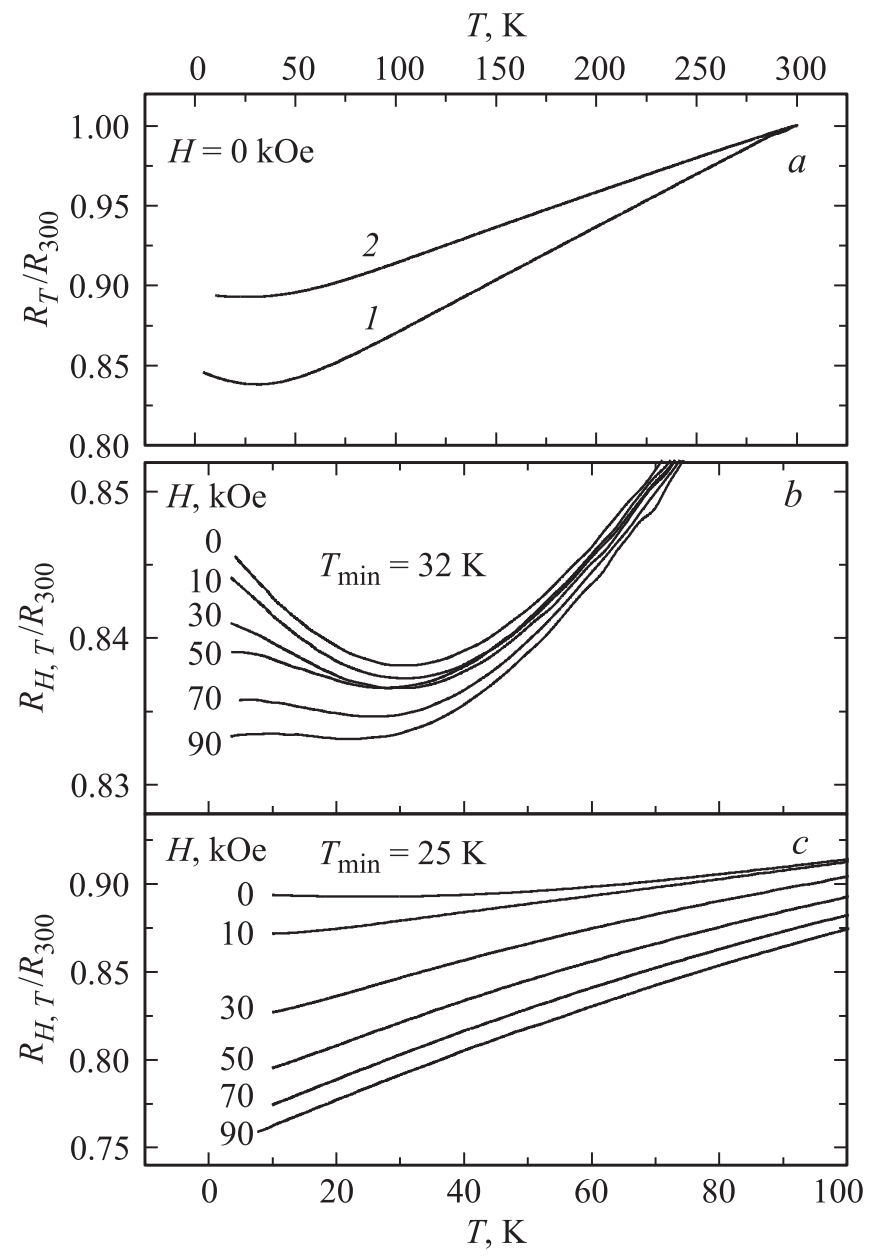

Рис. 9. Температурная зависимость электросопротивления при $H=0$ сверхрешеток $\mathrm{Co}\left(t_{x}, \AA\right) / \mathrm{Cu}(9.6 \AA)\left(t_{x}, \AA: 1-0.3\right.$, $2-1)(a)$ и в магнитном поле до $90 \mathrm{kOe}: t_{\mathrm{Co}}=0.3 \AA(b)$, $t_{\mathrm{Co}}=1 \AA(c)$.

электросопротивления, заключающаяся в том, что с понижением температуры электросопротивление наноструктуры сначала уменьшается, а затем, начиная с некоторой критической температуры, увеличивается, т. е. имеет место смена знака температурного коэффициента сопротивления. Измерения показали, что минимум на кривой $\rho(T)$ у наноструктуры $\mathrm{Co}(0.3 \AA) / \mathrm{Cu}(9.6 \AA)$ проявляется при $T=32 \mathrm{~K}$, а у наноструктуры с толщиной слоев кобальта $t_{\text {Со }}=1 \AA$ при $T=25 \mathrm{~K}$. На рис. $9, b$ и $c$ представлены кривые электросопротивления данных образцов при наложении внешнего магнитного поля до $90 \mathrm{kOe}$. Видно, что при увеличении напряженности магнитного поля происходит подавление температурной аномалии электросопротивления. Подобное поведение низкотемпературного электросопротивления при наложении магнитного поля характерно для сплавов, обладающих эффектом Кондо. Отметим, что кондоподобное поведение электросопротивления наблюдалось в микропроволоках состава $\mathrm{Co}_{5} \mathrm{Cu}_{95}$ в работе [26]. Отметим также, что в сверхрешетках $\mathrm{Co} / \mathrm{Cu}$ аномальное поведение электросопротивления выражено значительно слабее, чем в сверхрешетках $\mathrm{Fe} / \mathrm{Cr}$ с ультратонкими слоями $\mathrm{Fe}$ [27].

\section{7. Заключение}

Исследованы магнитооптические, оптические и магнитотранспортные свойства сверхрешеток $\mathrm{Co}\left(t_{x}, \AA\right) / \mathrm{Cu}(9.6 \AA)$, отличающихся толщиной слоев Со.

На магнитооптических кривых $\omega \operatorname{Im} \sigma_{x y}(\omega)$ сверхрешеток со слоями $t_{\text {Со }}=3-15 \AA$ в УФ области спектра $(\hbar \omega=5-6.2 \mathrm{eV})$ обнаружена предсказанная теоретически [21] полоса осцилляционного типа („петля“), обусловленная обменным расщеплением $3 d$-зоны $f c c$ Со $\left(2 \Delta_{e x}^{3 d} \sim 1 \mathrm{eV}\right)$.

Анализ толщинной зависимости времени релаксации электронов проводимости $\tau_{\mathrm{opt}}^{\mathrm{eff}}\left(t_{\mathrm{Co}}\right)$ сверхрешеток позволяет выделить три участка толщин слоя Со. Область (1) $\left(t_{\text {Со }}=7-15 \AA\right)$ - сверхрешетки со сплошными FM слоями и высокими значениями $\tau_{\mathrm{opt}}^{\mathrm{eff}}=(4.9-5.9) \cdot 10^{-15} \mathrm{~s}$. Область $(2) \quad\left(t_{\mathrm{Co}}=2-3 \AA\right)-$ кластерно-слоистые наноструктуры с FM-, SPM-частицами и парамагнитными включениями Со. В этой области при толщине слоя Со $3 \AA$ нами обнаружена смена знака ЭЭК на большом участке спектра при сохранении величины эффекта. Численный анализ ЭЭК в рамках гранулярной модели позволяет констатировать, что смена знака экваториального эффекта Керра обусловлена перераспределением атомов Со между SPM, FM и парамагнитной фракциями в пользу последней, что приводит к потере FM составляющей в магнитооптическом отклике мультислойной пленки с $t_{\text {Со }}=2 \AA$. Кластерно-слоистые наноструктуры с толщиной слоя Со 2 и $3 \AA$ имеют самые низкие значения времени релаксации $\tau_{\mathrm{opt}}^{\mathrm{eff}}=1.02 \cdot 10^{-15} \mathrm{~s}$ и $\tau_{\mathrm{opt}}^{\mathrm{eff}}=1.2 \cdot 10^{-15} \mathrm{~s}$ соответственно. Резкий спад $\tau_{\mathrm{opt}}^{\mathrm{eff}}$ между толщинами слоя Со 7 и $3 \AA$ характеризует промежуточную область кластеризации слоев кобальта. Дальнейшее измельчение кластеров Со в интерфейсных областях и снижение их концентрации в матрице $\mathrm{Cu}$ при толщинах $t_{\mathrm{Co}}=0.3-1 \AA$ вызывает рост функции $\tau_{\mathrm{opt}}^{\mathrm{eff}}$ (область 3). В этой металлической среде наблюдается аномальная температурная зависимость электросопротивления $\rho(T)$, которая исчезает при наложении магнитного поля $H$, подобно тому как это происходит в сплавах, обладающих эффектом Кондо.

Структурные исследования на рентгеновском дифрактометре PANalytical Empyrean, магнитные измерения в полях до $50 \mathrm{kOе}$ и измерение электросопротивления при низких температурах и в магнитных полях до $90 \mathrm{kOe}$ выполнены в ЦКП „Испытательный центр нанотехнологий и перспективных материалов“ ИФМ УрО РАН.

Авторы признательны Н.Н. Сидун за помощь в графическом оформлении результатов экспериментальных исследований. 


\section{Список литературы}

[1] S.S.P. Parkin, R. Bhadra, K.P. Roche. Phys. Rev. Lett. 66, 2152 (1991).

[2] S.S.P. Parkin, Z.G. Li, D. J. Smith. Appl. Phys. Lett. 58, 2710 (1991).

[3] T.N. Todorov, E.Yu. Tsymbal, D.G. Pettifor. Phys. Rev. B 54, R12685 (1996).

[4] E.Y. Tsymbal, D.G. Pettifor. Phys. Rev. B 54, 15314 (1996).

[5] W.P. Pratt, jr., S.F. Lee, P. Holody, Q. Yang, R. Lololee, J. Bass, P.A. Schroeder. J. Magn. Magn. Mater. 126, 406 (1993).

[6] L. Piraux, S. Dubois, C. Marchal, J.M. Beuken, L. Filipozzi, J.F. Despres, K. Ounadjela, A. Fert. J. Magn. Magn. Mater. 156, 317 (1996).

[7] I.D. Lobov, M.M. Kirillova, A.A. Makhnev, M.A. Milyaev, L.N. Romashev, V.V. Ustinov. J. Magn. Magn. Mater. 389, 169 (2015).

[8] M.D. Stiles, D.R. Penn. Phys. Rev. B 61, 3200 (2000).

[9] V.K. Sankaranarayann, O. Prakash, S.T. Lakshmikumar. J. Surf. Sci. Technol. 22, 15 (2006).

[10] J. Balogh, M. Csontos, D. Kaptás, G. Miháby. Sold State Commun. 126, 427 (2003).

[11] F. Spizzo, E. Angeli, D. Bisero, P. Vavassori, F.Ronconi. Appl. Phys. Lett. 79, 3293 (2001).

[12] I. Bakonyi, L. Péter, Z. Rolik, K. Kiss-Szabó, Z. Kupay, J. Tóth, L.F. Kiss, J. Pádár. Phys. Rev. B 70, 054427 (2004).

[13] P. Allia, P. Tiberto, F. Vinai, L. Pareti, G. Turilli. J. Magn. Magn. Mater. 196, 56 (1999).

[14] E. Jedryka, M. Wójcik, S. Nadolski, D.J. Kubinski, H. Holloway. J. Magn. Magn. Mater. 165, 292 (1997).

[15] Д.Л. Халяпин, П.Д. Ким, J. Kim, И.А. Турпанов, А.Я. Бетенькова, Г.В. Бондаренко, Т.Н. Исаева, І. Кіт. ФТТ 52 , 1665 (2010).

[16] В.М. Маевский. Деп. № 2461-В93. ВИНИТИ, М. (1993). $79 \mathrm{c}$

[17] T.K. Xia, P.M. Hia, D. Stroud. J. Appl. Phys. 67, 2736 (1990).

[18] Г.С. Кринчик. Физика магнитных явлений. Изд. МГУ, М. (1985). $336 \mathrm{c}$

[19] Г.А. Болотин, В.М. Маевский. ФММ 30, 475 (1970).

[20] E. Ganshina, A. Granovsky, V. Gushin, M. Kuzmichev, P. Podrugin, A. Kravetz, E. Shipil. Phys. A 241, 45 (1997).

[21] Ю.А. Успенский, С.В. Халилов. ЖЭТФ 95, 1022 (1989).

[22] Г.С. Кринчик, В.А. Артемьев. ЖЭТФ 53, 1901 (1967).

[23] K. Nakajima, T. Miyazaki. J. Appl. Phys. 79, 8, 4977 (1996).

[24] R. Castaner, C. Prieto, A de Andrés, J.L. Martinez, J.L. Martinez-Albertos, C. Ocal, R. Miranda. J. Phys.: Condens. Matter. 6, 4981 (1994).

[25] C.A. Chang. J. Magn. Magn. Mater. 109, 243 (1992).

[26] V. Zhukova, J.J. del Val, M. Ipatov, M. Ilyn, A. Granovsky, A. Zhukov. Proc. 8th Int. Conf. Sensing Technology. Liverpool, UK (2014). P. 332.

[27] V.V. Ustinov, L.N. Romashev, M.A. Milyaev, A.V. Korolev, T.P. Krinitsina, A.M. Burkhanov. J. Magn. Magn. Mater. 300, 148 (2006). 